\title{
GENERALIZED PENTAGONAL GEOMETRIES
}

\author{
A. D. FORBES AND C. G. RUTHERFORD
}

\begin{abstract}
A pentagonal geometry $\operatorname{PENT}(k, r)$ is a partial linear space, where every line is incident with $k$ points, every point is incident with $r$ lines, and for each point $x$, there is a line incident with precisely those points that are not collinear with $x$.

Here we generalize the concept by allowing the points not collinear with $x$ to form the point set of a Steiner system $S(2, k, w)$ whose blocks are lines of the geometry.
\end{abstract}

\section{INTRODUCTION}

A Steiner system $S(2, k, w)$ is an ordered pair $(W, \mathcal{B})$ such that (i) $W$ is a set of points with $|W|=w$, (ii) $\mathcal{B}$ is a set of $k$-subsets of $B$, called blocks, and (iii) each pair $\{x, y\} \subseteq W$ is a subset of precisely one block of $\mathcal{B}$.

A partial linear space is uniform if every line is incident with the same number of points, and regular if every point is incident with the same number of lines. For the purpose of this paper, a generalized pentagonal geometry is a uniform, regular partial linear space such that for each point $x$, there is a Steiner system $\left(W_{x}, \mathcal{B}_{x}\right)$ where $W_{x}$ is the set of points which are not collinear with $x$ and the blocks of $\mathcal{B}_{x}$ are lines of the geometry. By uniformity and regularity the number of points that are collinear with $x$ does not depend on $x$. Therefore $\left|W_{x}\right|$ must also be constant.

If $k$ is the number of points incident with a given line, $r$ is the number of lines incident with a given point and $w$ is the point set size of the Steiner systems, then $k, r$ and $w$ are constants, and we denote a generalized pentagonal geometry with these parameters by $\operatorname{PENT}(k, r, w)$. The Steiner system $S(2, k, w)$ consisting of the points that are not collinear with point $x$ is called the opposite design to $x$ and is denoted by $x^{\text {opp }}$. The notation $x^{\text {opp }}$ may refer to the $S(2, k, w)$ itself, or its point set, or its block set, whichever is appropriate from the context.

To avoid excessive trivialities we always assume that $k \geq 2$. Also we recognise three simplistic cases: for all $k \geq 2$, there exist Steiner systems $S(2, k, 0), S(2, k, 1)$ and $S(2, k, k)$, with block set sizes 0,0 and 1 respectively. If a Steiner system $S(2, k, w)$ exists, then the number of blocks is $w(w-$ $1) /(k(k-1))$ and the number of blocks that contain a given point is a

2010 Mathematics Subject Classification. 05B25.

Key words and phrases. pentagonal geometry, generalized pentagonal geometry, group divisible design. 
constant, $(w-1) /(k-1)$. An $S(2,2, w)$ is essentially a complete graph $K_{w}$, and an $S(2,3, w)$ is also called a Steiner triple system of order $w, \operatorname{STS}(w)$. An affine plane of order $n$ is a Steiner system $S\left(2, n, n^{2}\right)$ and a projective plane of order $n$ is a Steiner system $S\left(2, n+1, n^{2}+n+1\right)$. We also refer to Steiner systems generally as designs. A necessary and sufficient condition for the existence of a Steiner system $S(2, k, w)$ with $w \geq 1$ is $w \equiv 1$ or $k(\bmod k(k-$ 1)) when $k=2$ (trivially), $k=3$, [21], and $k \in\{4,5\},[18]$. An affine plane and a projective plane of order $n$ exist whenever $n$ is a prime power.

A $\operatorname{PENT}(k, r, 0)$ is essentially a Steiner system $S(2, k,(k-1) r+1)$, and a $\operatorname{PENT}(k, r, 1)$ consists of the points and blocks of a $k$-GDD of type $2^{r(k-1) / 2+1}$ as defined in Section 2. If $w=k$, the structure is known as a pentagonal geometry and may be denoted by $\operatorname{PENT}(k, r)$. We must have $r \geq(w-1) /(k-1)$ and, as previously stated, we always assume $k \geq 2$. For the remainder of the paper, we assume $w \geq k$ in order to avoid $S(2, k, w)$ designs with empty block sets, and wherever necessary we tacitly assume that the relevant Steiner systems exist.

For convenience, we shall regard lines as sets of points - thus two points are collinear if they are on (i.e. elements of) the same line. Lines are also called blocks, and we refer to the parameter $k$ of a $\operatorname{PENT}(k, r, w)$ as the block size.

The deficiency graph of a generalized pentagonal geometry $\operatorname{PENT}(k, r, w)$ has as its vertices the points of the geometry, and there is an edge $x \sim y$ precisely when $x$ and $y$ are not collinear. It is clear that the graph must be $w$-regular and triangle-free.

For brevity, we adopt the notation $(w, g)$-graph for a graph that is $w$ regular and has girth $g$, and $\left(w, g^{+}\right)$-graph for a graph that is $w$-regular and has girth at least $g$. Thus we can say that a $\operatorname{PENT}(k, r, w)$ has a deficiency $\left(w, 4^{+}\right)$-graph.

The concept of a pentagonal geometry was introduced in [4] to provide a generalization of the pentagon analogous to the generalization of the polygon as described in [24] and [11]. The geometry described by Ball, Bamberg, Devillers \& Stokes in [4] is based on the observation that for each vertex $x$ of a pentagon, the two vertices that are not collinear with $x$ form a line. According to their definition the pentagon is a $\operatorname{PENT}(2,2)$.

Our generalization is based on the observation that a line containing $k$ points is the single block of the Steiner system $S(2, k, k)$. It seems natural, therefore, to extend the definition of $x^{\text {opp }}$ to a general Steiner system $S(2, k, w)$ since these designs have the same relevant property as a single line: any two points are collinear. According to our definition the pentagon is a $\operatorname{PENT}(2,2,2)$. For a more substantial example, let $H$ be the HoffmanSingleton graph, the unique 50-vertex $(7,5)$-graph, [19]. Then we know that the neighbours of the vertices of $H$ form the lines of a $\operatorname{PENT}(7,7)$, [4, Section 3]. For each vertex $x$ of $H$, construct a Steiner triple system of order 7 on the set of neighbours of $x$. Since the neighbourhoods of two distinct vertices of $H$ cannot have more than one common vertex-otherwise the graph 
would not have girth 5 - the block sets of the fifty STS(7)s are mutually disjoint. The 350 blocks so obtained are the lines of a $\operatorname{PENT}(3,21,7)$. We are also motivated by the limitations of our knowledge concerning pentagonal geometries $\operatorname{PENT}(k, r)$ with $k>7$. In Section 3 we see that it is not especially difficult to find examples of $\operatorname{PENT}(3, r, w)$ with connected deficiency $\left(w, 5^{+}\right)$-graphs for $w \in\{9,13,15,19,21\}$ but we are not aware of any examples of the corresponding $\operatorname{PENT}(w, r)$.

If $w \geq k \geq 2$ and there exists an $S(2, k, w)$, then there exists a $\operatorname{PENT}(k,(w-1) /(k-1), w)$. In this generalized pentagonal geometry, which by analogy with [4] we regard as degenerate, the lines are the blocks of two Steiner systems $S(2, k, w)$ with disjoint point sets and the deficiency graph is a complete bipartite graph $K_{w, w}$. Thus for any point $x$ in either one of the Steiner systems, $x^{\text {opp }}$ is the other system. The lines of a degenerate geometry can also occur in the line set of a larger generalized pentagonal geometry, and, again by analogy with [4], we refer to the substructure as an opposite design pair, or when $w=k$, an opposite line pair.

For the general theory of pentagonal geometries as well as further background material, we refer the reader to [4]. The subject was further developed in [17], [14] and [12]. The existence spectrum was settled for $\operatorname{PENT}(2, r)$ in [4, Corollary 2.6], and for $\operatorname{PENT}(3, r)$ as follows: unconditionally, i.e. with no restriction on the number of opposite line pairs, $[17$, Theorem 9]; for geometries without opposite line pairs, [14]; and for geometries with a given number of opposite line pairs, [14]. Similar results have been established for $\operatorname{PENT}(4, r)$ : unconditionally, with a few possible exceptions, [14]; for geometries without opposite line pairs, with a few possible exceptions, [12]; and for a given number of opposite line pairs and sufficiently large $r,[12]$. Reference [12] also deals with block size 5, where some progress is made towards the solution of the existence spectrum problem for $\operatorname{PENT}(5, r)$ without opposite line pairs. If opposite line pairs are allowed, the spectrum for $\operatorname{PENT}(5,5 t+1)$ was determined up to a few possible exceptions in [17, Theorem 12].

The situation regarding pentagonal geometries $\operatorname{PENT}(k, r)$ with connected deficiency graphs is far from resolved for $k \geq 3$. All of the construction methods employed in [17], [14] and [12] use group divisible designs, as in Theorem 2.5, below, and inevitably produce geometries with deficiency graphs consisting of at least $k$ connected components. As far as we are aware, the known $\operatorname{PENT}(k, r)$ with $k \geq 3$ and connected deficiency graph of girth at least 5 amount to the following:

(i) a small number of $\operatorname{PENT}(3, r)$ constructed by hand, including the Desargues configuration, $\operatorname{PENT}(3,3)$, [4];

(ii) $\operatorname{PENT}(3, r)$ that can be created by hill climbing for $r$ not too large, [14];

(iii) $\operatorname{PENT}(3, r)$ for all $r \equiv 3(\bmod 6), r \geq 33$, [12]; 
(iv) $\operatorname{PENT}(4, r)$ for $r \in\{13,17,20,21,24,29,33,37,40,45,49,52,53$, $60,61,65,69,77,80,81,85,93,97,100,101,108,109,117,120$, $125,133,140,141,149,157,160,165,173,180\},[12]$;

(v) $\operatorname{PENT}(5, r)$ for $r \in\{20,25,30,35,40\}$, [12];

(vi) the $\operatorname{PENT}(6,7)$ and $\operatorname{PENT}(7,7)$ based on the Hoffman-Singleton graph, [4].

In the next section we describe the basic properties of generalized pentagonal geometries, relating them to the corresponding results of [4]. We find that although many of the lemmas in [4, Section 2] concerning pentagonal geometries transfer straightforwardly to $\operatorname{PENT}(k, r, w)$, there are some properties that do not. For example, when $r>(w-1) /(k-1)$ a $\operatorname{PENT}(k, r, w)$ can have a connected deficiency graph with girth 4 whereas this is impossible if $w=k$. We also settle the existence problem for $\operatorname{PENT}(2, r, w)$. In Section 3 we show how to construct $\operatorname{PENT}(3, r, w)$ geometries with deficiency $\left(w, 5^{+}\right)$-graphs, and we prove that these exist for $w \in\{7,9,13,15$, $19,21,25,27,31,33\}$ and all sufficiently large admissible $r$. In Section 4 we present a number of $\operatorname{PENT}(4, r, 13)$ geometries with connected deficiency $\left(13,5^{+}\right)$-graphs. Consequently we show that a $\operatorname{PENT}(4, r, 13)$ with deficiency $\left(13,5^{+}\right)$-graph exists for every sufficiently large even $r$.

\section{Generalized pentagonal geometries}

Lemma 2.1. A generalized pentagonal geometry $\operatorname{PENT}(k, r, w)$ has $r(k-$ $1)+w+1$ points and $(r(k-1)+w+1) r / k$ lines. $A \operatorname{PENT}(k, r, w)$ exists only if $r(r-w-1) \equiv 0(\bmod k)$.

Proof. Consider a point, $x$. Every other point is either on one of the $r$ lines that contain $x$ or is one of the $w$ points of $x^{\text {opp }} ; r(k-1)+w+1$ points altogether.

Let $v=r(k-1)+w+1$. The number of incident point-line pairs is $v r$. However, each line contains $k$ points. Therefore the number of lines is $v r / k$, which must of course be an integer.

Given $k$ and $w$, we say that $r$ is admissible if $r(w+1-r) \equiv 0(\bmod k)$.

Lemma 2.2. If $x$ and $y$ are distinct points in a generalized pentagonal geometry such that there exist two distinct points common to both $x^{\mathrm{opp}}$ and $y^{\mathrm{opp}}$, then $x^{\mathrm{opp}}$ and $y^{\mathrm{opp}}$ have a common block.

Proof. If $a$ and $b$ are points in both $x^{\text {opp }}$ and $y^{\text {opp }}$, then the block containing $a$ and $b$ must belong to both opposite designs.

In [4, Lemma 2.3] it is shown that if $x$ and $y$ are distinct points of a $\operatorname{PENT}(k, r)$ such that $x^{\mathrm{opp}}=y^{\mathrm{opp}}=L$, then there exists a unique opposite line pair $\{L, M\}$ such that $x, y \in M$. However, this does not generally extend to geometries $\operatorname{PENT}(k, r, w)$ where $w>k$. For example, take the $\operatorname{PENT}(2,11,8)$ shown in Figure 1, which is clearly a special case of a general construct, $\operatorname{PENT}(2,3 m-1,2 m)$. Here we have five copies of $K_{4}$, represented 
as the 'spokes', linked by the edges of five copies of $K_{4,4}$ and, for instance, $1^{\text {opp }}=2^{\text {opp }}=3^{\text {opp }}=4^{\text {opp }}=\{9,10, \ldots, 16\}$ whereas $x^{\text {opp }} \neq y^{\text {opp }}$ for $x \in$ $\{9,10,11,12\}, y \in\{13,14,15,16\}$. Observe also that the deficiency graph is connected, is 8-regular and has girth 4 .

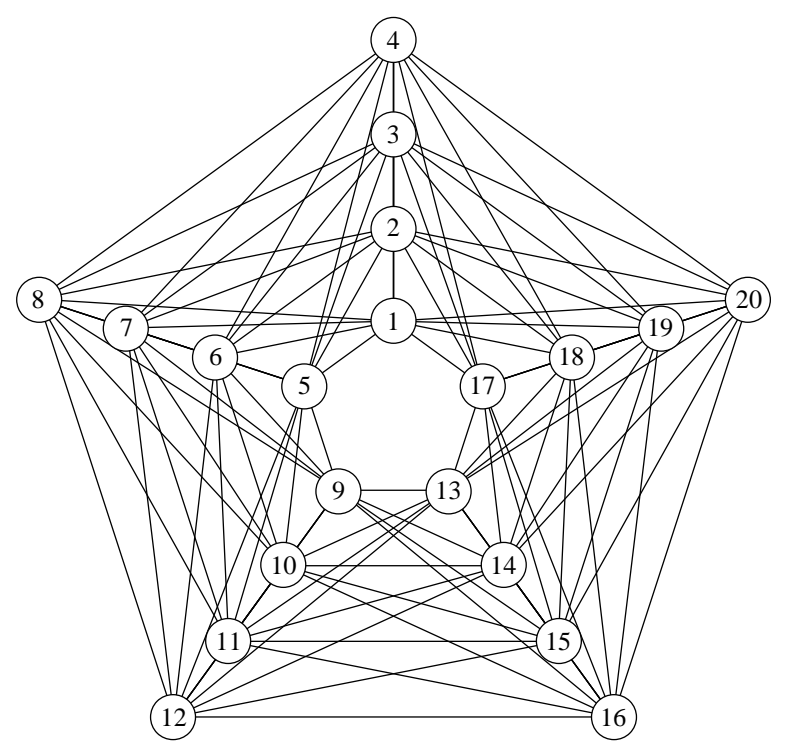

Figure 1. $\operatorname{PENT}(2,3 m-1,2 m), m=4$

Lemma 2.3. Let $D$ be the deficiency graph of a generalized pentagonal geometry $\operatorname{PENT}(k, r, w)$. Then $D$ is w-regular and has girth at least 4 .

For distinct points $x$ and $y$, let $U_{x, y}$ denote the set of points common to both $x^{\mathrm{opp}}$ and $y^{\mathrm{opp}}$, and let $u_{x, y}=\left|U_{x, y}\right|$. Then $U_{x, y}$ is the point set of a Steiner system $S\left(2, k, u_{x, y}\right)$ that exists as a subdesign of both $x^{\text {opp }}$ and $y^{\text {opp }}$.

Proof. For any vertex $x$ of $D$, the neighbourhood of $x$ is the point set of $x^{\text {opp}}$, which has cardinality $w$. If $D$ contains a path, $a \sim x \sim b$, then $a$ and $b$ are in $x^{\text {opp }}$ and must therefore be collinear. Hence $D$ is triangle-free. The neighbours of vertices $x$ and $y$ in $D$ are clearly the point sets of $x^{\text {opp }}$ and $y^{\text {opp }}$ respectively.

When $u_{x, y}=0$ it follows vacuously that an $S(2, k, 0)$ is a subdesign of both $x^{\text {opp }}$ and $y^{\text {opp }}$. If $u_{x, y}=1$, then $U_{x, y}$ is clearly the point set of an $S(2, k, 1)$ which is a subdesign of both $x^{\text {opp }}$ and $y^{\text {opp }}$.

Suppose $u_{x, y} \geq 2$ and note that the points of $U_{x, y}$ are pairwise collinear. Hence, for any pair of distinct points $a$ and $b$, there is a line containing both $a$ and $b$ that must belong to the line set of both $x^{\text {opp }}$ and $y^{\text {opp }}$. Let $\mathcal{L}$ be the set of all these lines. Then $\left(U_{x, y}, \mathcal{L}\right)$ is a Steiner system $S\left(2, k, u_{x, y}\right)$, and $\mathcal{L}$ is contained in the line sets of both $x^{\text {opp }}$ and $y^{\text {opp }}$. 
The possible values of $u_{x, y}$ in Lemma 2.3 always include $0,1, k$ and $w$, and by Fisher's inequality for block designs, if $w>k$, then $w \geq k^{2}-k+1$. Clearly we cannot have $1<u_{x, y}<k$ whereas for values strictly between $k$ and $w$, it depends on whether there exists a Steiner system $S\left(2, k, u_{x, y}\right)$ embedded in both of the Steiner systems $S(2, k, w)$ that form $x^{\mathrm{opp}}$ and $y^{\mathrm{opp}}$. For example, in a $\operatorname{PENT}(3, r, 19)$ where the $\operatorname{STS}(19)$ s used for the opposite designs include subdesigns $\operatorname{STS}(7)$ and $\operatorname{STS}(9)$, [8, Table 4], one can have $u_{x, y} \in\{0,1,3,7,9,19\}$.

Lemma 2.4. For a generalized pentagonal geometry $\operatorname{PENT}(k, r, w)$, the following properties are equivalent:

(i) for any two distinct points $x$ and $y, x^{\text {opp }}$ and $y^{\text {opp }}$ have no common block;

(ii) for any two distinct points $x$ and $y$, the point sets of $x^{\mathrm{opp}}$ and $y^{\mathrm{opp}}$ have at most one common point;

(iii) the deficiency graph has girth at least 5 .

Proof. (i) $\Rightarrow$ (ii): Suppose for distinct points $x$ and $y, x^{\text {opp }}$ and $y^{\text {opp }}$ have distinct common points $a$ and $b$. Then there exists a unique line containing $a$ and $b$ that must be present in the line sets of both $x^{\text {opp }}$ and $y^{\text {opp }}$.

(ii) $\Rightarrow$ (iii): We know from Lemma 2.3 that the deficiency graph has girth at least 4 . Suppose the deficiency graph contains a 4-cycle, $(x, a, y, b)$. Then $a$ and $b$ are in both $x^{\text {opp }}$ and $y^{\text {opp }}$.

(iii) $\Rightarrow$ (i): Suppose for distinct points $x$ and $y, x^{\text {opp }}$ and $y^{\text {opp }}$ have a common block containing points $a$ and $b$. Then the deficiency graph contains a 4-cycle, $(x, a, y, b)$.

For clarification in the rest of the paper, we define the property pairwise disjoint opposite designs of a generalized pentagonal geometry to mean that for any two distinct points $x$ and $y, x^{\text {opp }}$ and $y^{\text {opp }}$ have no common block, i.e. (i) of Lemma 2.4. Thus we are referring to the whole collection of opposite designs, including possible repetitions, and we allow two opposite designs to have at most one common point. As we have seen, the property is equivalent to having a deficiency graph with girth at least 5 .

Lemma 2.5. For a $\operatorname{PENT}(k, r, w)$ with deficiency graph of girth at least 5 , we have $r \geq w(w-1) /(k-1)$.

Proof. Let $v$ be the number of points in the $\operatorname{PENT}(k, r, w)$. An opposite design contains $w(w-1) /(k(k-1))$ blocks. Since the opposite designs are pairwise disjoint by Lemma 2.4, the number of blocks, $v r / k$, cannot be less than $v w(w-1) /(k(k-1))$.

When equality occurs in Lemma 2.5 the number of points is $v=w^{2}+1$, the Moore bound for $(w, 5)$-graphs.

Lemma 2.6. Let $x$ and $y$ be two distinct points in a generalized pentagonal geometry with deficiency graph $D$. Then the distance in $D$ between $x$ and $y$ 
is 2 if and only if there exists a point $z$ such that $z^{\text {opp }}$ contains both $x$ and $y$.

Proof. This is similar to [4, Lemma 2.7]. Suppose $x$ and $y$ are distinct points. If $x$ and $y$ are at distance 2 in $D$, then $x \not y$ and there exists a point $z$ such that $x \sim z \sim y$; hence $x$ and $y$ belong to $z^{\text {opp }}$. On the other hand, if $x$ and $y$ belong to $z^{\text {opp }}$, then there is a line in $z^{\text {opp }}$ containing both $x$ and $y$; therefore $x \sim z \sim y$ and $x \not y$.

Thus the deficiency graph of a $\operatorname{PENT}(k, r, w)$ with pairwise disjoint opposite designs has $r(k-1)+w+1$ vertices and is a $\left(w, 5^{+}\right)$-graph. Also the distance between vertices $x$ and $y$ is at least 3 if $x$ and $y$ are distinct points on the same line that is not part of an opposite design.

Theorem 2.1. Suppose $w \geq 2$. A generalized pentagonal geometry $\operatorname{PENT}(2, r, w)$ exists if and only if there exists a $w$-regular graph with $r+w+1$ vertices and girth at least 4. A generalized pentagonal geometry $\operatorname{PENT}(2, r, w)$ with pairwise disjoint opposite designs exists if and only if there exists a $w$-regular graph with $r+w+1$ vertices and girth at least 5 .

Proof. Let $v=r+w+1$ and suppose $D$ is a $v$-vertex $\left(w, 4^{+}\right)$-graph. The edges of the complement of $D$ are clearly the $v(v-1) / 2-w v / 2=v r / 2$ blocks of a $\operatorname{PENT}(2, r, w)$ in which for each point $x$, the neighbours of vertex $x$ in $D$ comprise $x^{\text {opp }}$. Now suppose in addition that $D$ has girth at least 5 . If there exist points $x$ and $y$ such that $x^{\text {opp }}$ and $y^{\text {opp }}$ have a common a block, $\{a, b\}$ say, then there is a 4 -cycle in $D,(x, a, y, b)$. Hence the opposite designs must be pairwise disjoint.

Conversely, by Lemmas 2.3 and 2.4, the deficiency graph of a $\operatorname{PENT}(2, r, w)$ has the stated properties.

If $w=k$ and $x^{\text {opp }}=y^{\text {opp }}$ for distinct points $x$ and $y$, then $x^{\text {opp }}$ and the line containing $\{x, y\}$ must form an opposite line pair, [4, Lemma 2.3]. On the other hand, when $w>k$ the situation is not so simple. As we have seen, it is possible to have distinct points $x, y$ such that $x^{\text {opp }}$ and $y^{\text {opp }}$ contain a common block but do not form an opposite design pair. We leave the investigation of this complication as well as a detailed discussion of geometries containing opposite design pairs for a possible future paper. Henceforth we shall confine our attention mainly to generalized pentagonal geometries where the opposite designs are pairwise disjoint, or equivalently by Lemma 2.4, where the deficiency graph has girth at least 5 . Now pairs of vertices of the deficiency graph of a $\operatorname{PENT}(k, r, w)$ with $v=(k-1) r+w+1$ may be categorized according to their contribution to the geometry:

(i) $v w / 2$ adjacent pairs, which necessarily have no common neighbour, are not collinear points in the geometry;

(ii) $w(w-1) v / 2$ non-adjacent pairs with one common neighbour are collinear in the blocks of the opposite designs;

(iii) $\left(v-w^{2}-1\right) v / 2$ non-adjacent pairs with no common neighbour are collinear in the blocks that do not occur in opposite designs. 
Another way of looking at a $\operatorname{PENT}(k, r, w)$ is given by the next theorem.

Theorem 2.2. Let $k \geq 2, w \geq k$ and $r \geq w(w-1) /(k-1)$ be integers. Let $v=(k-1) r+w+1$ and $V=\{1,2, \ldots, v\}$. Then there exists a pentagonal geometry $\operatorname{PENT}(k, r, w)$ with point set $V$ and having a deficiency graph of girth at least 5 if and only if there exists an edgewise decomposition of the complete graph $K_{v}$ with vertex set $V$ into the following graphs, each with vertex set $V$ :

(i) a w-regular graph, $D$, with girth at least 5;

(ii) a w(w-1)-regular graph $S$ which itself has an edgewise decomposition into $v$ complete graphs $K_{w}, S_{1}, S_{2}, \ldots, S_{v}$, say, such that for $i=1$, $2, \ldots, v$, the vertex set of $S_{i}$ is the neighbourhood set of vertex $i$ of $D$, and $S_{i}$ admits an edgewise decomposition into $w(w-1) /(k(k-1))$ complete graphs $K_{k}$;

(iii) if $r>w(w-1) /(k-1), a\left(v-w^{2}-1\right)$-regular graph, $T$, which admits an edgewise decomposition into $\left(v-w^{2}-1\right) v /(k(k-1))$ complete graphs $K_{k}$. Moreover, if $r=w(w-1) /(k-1)+1$, the graphs $K_{k}$ partition the vertex set of $T$.

Proof. A Steiner system $S(2, k, w)$ exists if and only if there exists an edgewise decomposition of the complete graph $K_{w}$ into complete graphs $K_{k}$.

The neighbourhood set of vertex $x$ of $D$ is the point set of $x^{\text {opp }}$, and $x^{\text {opp }}$ is the $S(2, k, w)$ corresponding to $S_{x}$,

Since $D$ is a $\left(w, 5^{+}\right)$graph, two neighbourhood sets of $D$ cannot have more than one common vertex, and a given vertex $x$ of $D$ occurs in precisely $w$ of the graphs $S_{i}$, i.e. those corresponding to $\left\{y^{\mathrm{opp}}: y \in x^{\mathrm{opp}}\right\}$. Moreover, considered as a point in the Steiner system $S(2, k, w), x$ occurs in precisely $(w-1) /(k-1)$ of its blocks. Therefore $x$ occurs in precisely $w-1$ edges of those $S_{i}$ that contain $x$. Hence $S$ is $w(w-1)$-regular and $T$ is $(v-1-w-$ $w(w-1))$-regular. Also $T$ has an edgewise decomposition into $K_{k}$ graphs corresponding to the

$$
\frac{v}{k}\left(r-\frac{w(w-1)}{k-1}\right)=\frac{v}{k} \cdot \frac{v-w^{2}-1}{k-1}
$$

lines that do not belong to any opposite design. If $r=w(w-1) /(k-1)+1$, then $T$ is $(k-1)$-regular and has an edgewise decomposition into $v / k K_{k}$ graphs, which must therefore partition $V$.

Theorem 2.3. (i) There is no $\operatorname{PENT}(k, w(w-1) /(k-1), w)$ with deficiency graph of girth at least 5 if $w \geq k \geq 2$ and $w \notin\{2,3,7,57\}$.

(ii) Suppose $w \geq k \geq 2, w \in\{2,3,7\}$ and $r=w(w-1) /(k-1)$. Then there exists a $\operatorname{PENT}(k, r, w)$ with deficiency graph of girth 5 if and only if

$$
(k, r, w) \in\{(2,2,2),(2,6,3),(3,3,3),(2,42,7),(3,21,7),(7,7,7)\} .
$$

Proof. (i) The number of points is $w^{2}+1$, the Moore bound for $(w, 5)$-graphs. Hence a $\operatorname{PENT}(k, w(w-1) /(k-1), w)$ cannot exist unless $w$ has one of the stated values; see, for example, [7, Section 11.12]. 
(ii) The existence of a $\operatorname{PENT}(k, w(w-1) /(k-1), w)$ requires a Steiner system $S(2, k, w)$, and therefore the only possibilities are given by (1). For $(2,2,2),(3,3,3)$ and $(7,7,7)$, see $[4$, Section 3]. For $(2,6,3)$ take the Petersen graph and overlay the neighbourhood set of each point with a triangle. For $(2,42,7)$ and $(3,21,7)$, take the Hoffman-Singleton graph and overlay the neighbourhood set of each point with a $K_{7}$ or an STS(7), as appropriate.

If the 3250-vertex, 57-regular Moore graph with girth 5 exists, then so do generalized pentagonal geometries which have it as the deficiency graph: $\operatorname{PENT}(57,57,57),[4]$, as well as $\operatorname{PENT}(2,3192,57), \operatorname{PENT}(3,1596,57)$ and PENT $(8,456,57)$ obtained by overlaying the neighbourhood sets of the graph with complete graphs $K_{57}$, STS(57)s and projective planes of order 7 respectively.

Theorem 2.4. There is no $\operatorname{PENT}(k, w(w-1) /(k-1)+1, w)$ with deficiency graph of girth at least 5 for $w \geq k \geq 2$ except for $\operatorname{PENT}(2,3,2)$, $\operatorname{PENT}(6,7,6)$ and possibly $\operatorname{PENT}(56,57,56)$.

Proof. For $w=k$, see [4, Corollary 4.5].

Let $w>k \geq 2, r=w(w-1) /(k-1)+1$ and suppose there exists a $\operatorname{PENT}(k, r, w)$ with deficiency $\left(w, 5^{+}\right)$-graph. The number of points and the number of lines are respectively

$$
v=w^{2}+k \quad \text { and } \quad b=\frac{v r}{k}=\frac{\left(w^{2}+k\right)\left(w^{2}-w+k-1\right)}{k(k-1)} .
$$

Since the Moore bound for $(w, 6)$-graphs, namely $2\left(w^{2}-w+1\right)$, exceeds $w^{2}+k$ when $w>k \geq 2$, we may assume that the deficiency graph has girth 5 .

Suppose $k=2$. Then $w$ is even since the existence of the deficiency graph requires $v w$ to be even. In [6] it is proved that if $m$ is the smallest number of vertices for which a $(w, 5)$-graph exists, then $m \neq w^{2}+2$. Since $w>2$, the Moore bound, $w^{2}+1$, is not attained when $w$ is even. Consequently the smallest $(w, 5)$-graph has at least $w^{2}+3$ vertices. Hence there is no $\operatorname{PENT}(2, r, w)$ with $w>2$.

In the remainder of the proof we assume $k \geq 3$.

For $k=3$, it is known that there is no $(w, 5)$-graph with $w^{2}+3$ vertices except possibly when $w$ is even or when $w$ is odd and $w=\ell^{2}+\ell+1 \pm 2$ for some integer $\ell,[22]$. Although it would suffice to deal only with these exceptional $w$, it is convenient to include $k=3$ in our proof.

The number of lines in opposite designs is $v w(w-1) /(k(k-1))$ and therefore the number of non-opposite lines, i.e. lines that do not occur in opposite designs, is

$$
b_{\mathrm{rem}}=b-\frac{v w(w-1)}{k(k-1)}=\frac{v}{k}
$$


Hence $k \mid v$ and $k \mid w^{2}$. Moreover, if a Steiner system $S(2, k, w)$ exists, then $k(k-1) \mid w(w-1)$ and $k-1 \mid w-1$. It follows that $k \mid w$ and

$$
w=t k(k-1)+k \text { for some positive integer } t \text {. }
$$

Let $D$ denote the deficiency graph and let $E$ denote the distance at least 3 graph, where the vertices of $E$ are points of the geometry and there is an edge $i \sim j$ whenever the distance between $i$ and $j$ in $D$ is at least 3. Let $A$ and $B$ be the adjacency matrices of $D$ and $E$ respectively and denote the distance in $D$ between vertices $i$ and $j$ by $d(i, j)$. Then, recalling that $\left[A^{2}\right]_{i, j}$ is the number of 2 -step walks from vertex $i$ to vertex $j$, in row $i$ of $A^{2}$ we have

$$
\left\{\begin{array}{lll}
{\left[A^{2}\right]_{i, i}} & =w, & \text { which occurs once } \\
{\left[A^{2}\right]_{i, j, i \sim j}} & =0, & \text { which occurs } w \text { times } \\
{\left[A^{2}\right]_{i, j, d(i, j)=2}=1,} & \text { which occurs } w(w-1) \text { times } \\
{\left[A^{2}\right]_{i, j, d(i, j) \geq 3}=0,} & \text { which occurs } k-1 \text { times. }
\end{array}\right.
$$

Let $I, J$ and $\mathbf{j}$ denote the identity matrix, the all-ones square matrix and the all-ones vector respectively, each of dimension $v$. From (3) it is plain that $E$ is $(k-1)$-regular and

$$
B=J-A^{2}-A+(w-1) I .
$$

By Lemma 2.6, two distinct vertices of $E$ are adjacent precisely when they are on the same non-opposite line. Hence $E$ consists of $b_{\text {rem }}=v / k$ pairwise disjoint complete graphs $K_{k}$. Since $D$ is $w$-regular and is too small to have more than one component, $A$ has eigenvalue $w$ with multiplicity 1 and corresponding eigenvector $\mathbf{j}$. Let $\mathbf{s}$ be an eigenvector of $A$ that is orthogonal to $\mathbf{j}$ and let $s$ be its eigenvalue. Then from (4) we have

$$
B \mathbf{s}=\left(J-A^{2}-A+(w-1) I\right) \mathbf{s}=\left(-s^{2}-s+w-1\right) \mathbf{s},
$$

and hence $-s^{2}-s+w-1$ is an eigenvalue of $B$. But the eigenvalues of $B$ are $k-1$ and -1 . Therefore the eigenvalues of $A$ comprise:

(i) $w$ with multiplicity 1 and eigenvector $\mathbf{j}$;

(ii) roots of $-s^{2}-s+w-1=k-1$,

$$
s_{1}=\frac{-1+\sqrt{4 w-4 k+1}}{2}, s_{2}=\frac{-1-\sqrt{4 w-4 k+1}}{2},
$$

with multiplicities $m_{1}, m_{2}$ respectively;

(iii) roots of $-s^{2}-s+w-1=-1$,

$$
s_{3}=\frac{-1+\sqrt{4 w+1}}{2}, s_{4}=\frac{-1-\sqrt{4 w+1}}{2},
$$

with multiplicities $m_{3}, m_{4}$ respectively. 
Since $A$ is the adjacency matrix of a triangle-free graph, both $A$ and $A^{3}$ have zero trace. Moreover, by (3), the trace of $A^{2}$ is $v w$. We therefore have

$$
\begin{gathered}
1+\sum_{i=1}^{4} m_{i}=v, w^{2}+\sum_{i=1}^{4} m_{i} s_{i}^{2}=v w, v=w^{2}+k, \\
w+\sum_{i=1}^{4} m_{i} s_{i}=w^{3}+\sum_{i=1}^{4} m_{i} s_{i}^{3}=0,
\end{gathered}
$$

which has the solution

$$
\begin{aligned}
& m_{1}=\frac{w(w-2 k+w \sqrt{1-4 k+4 w})}{2 k \sqrt{1-4 k+4 w}}, \\
& m_{2}=\frac{w(2 k-w+w \sqrt{1-4 k+4 w})}{2 k \sqrt{1-4 k+4 w}}, \\
& m_{3}=\frac{(\sqrt{4 w+1}+1)\left(k^{2}+\left(w^{2}-1\right) k-w^{2}\right)}{2 k \sqrt{4 w+1}}, \\
& m_{4}=\frac{(\sqrt{4 w+1}-1)\left(k^{2}+\left(w^{2}-1\right) k-w^{2}\right)}{2 k \sqrt{4 w+1}} .
\end{aligned}
$$

By (2), we cannot have $w=2 k$ or $k^{2}+\left(w^{2}-1\right) k-w^{2}=0$; so if one of the eigenvalues is irrational, then at least one of the multiplicities is irrational.

Hence we may assume all eigenvalues of $A$ are rational and there exist integers $p$ and $q$ such that

$$
\sqrt{4 w-4 k+1}=2 p+1, \sqrt{4 w+1}=2 q+1 .
$$

Therefore

$$
w=p^{2}+p+k=q^{2}+q
$$

and it follows that $(q-p)(q+p+1)=k=d e$, say, where $d=q-p$ is a divisor of $k$. Then

and

$$
p=\frac{e-d-1}{2}, q=\frac{e+d-1}{2}
$$

$$
w-k=p^{2}+p=\frac{(d-e)^{2}-1}{4}<t k(k-1)=w-k,
$$

a contradiction.

For creating new generalized pentagonal geometries from existing ones, we use Theorem 2.5, below, a straightforward adaptation of Wilson's Fundamental Construction, [26], [16, Theorem IV.2.5]. For this theorem, the following definition is central.

A group divisible design, $k$-GDD, of type $g_{1}^{u_{1}} g_{2}^{u_{2}} \ldots g_{n}^{u_{n}}$ is an ordered triple $(V, \mathcal{G}, \mathcal{B})$ such that:

(i) $V$ is a base set of cardinality $u_{1} g_{1}+u_{2} g_{2}+\cdots+u_{n} g_{n}$;

(ii) $\mathcal{G}$ is a partition of $V$ into $u_{i}$ subsets of cardinality $g_{i}, i=1,2, \ldots, n$, called groups; 
(iii) $\mathcal{B}$ is a non-empty collection of $k$-subsets of $V$, called blocks; and

(iv) each pair of elements from distinct groups occurs in precisely one block but no pair of elements from the same group occurs in any block.

A Steiner system $S(2, k, w)$ is essentially a $k$-GDD of type $1^{w}$. A $k$-GDD of type $q^{k}$ is also called a transversal design, $\operatorname{TD}(k, q)$.

Theorem 2.5. Let $k \geq 2$ and $w \geq k$ be integers. For $i=1,2, \ldots, n$, let $r_{i}$ be a positive integer, let $v_{i}=(k-1) r_{i}+w+1$, and suppose there exists a generalized pentagonal geometry $\operatorname{PENT}\left(k, r_{i}, w\right)$. Suppose also that there exists a $k$-GDD of type $v_{1}^{u_{1}} v_{2}^{u_{2}} \ldots v_{n}^{u_{n}}$. Let $N=u_{1}+u_{2}+\cdots+u_{n}$ and $R=u_{1} r_{1}+u_{2} r_{2}+\cdots+u_{n} r_{n}$. Then there exists a generalized pentagonal geometry $\operatorname{PENT}(k, R+(N-1)(w+1) /(k-1), w)$.

Furthermore, if for $i=1,2, \ldots, n$, the $\operatorname{PENT}\left(k, r_{i}, w\right)$ has a deficiency graph of girth at least 5 , then so does the $\operatorname{PENT}(k, R+(N-1)(w+1) /(k-$ $1), w)$.

Proof. Overlay each group of size $v_{i}$ with a $\operatorname{PENT}\left(k, r_{i}, w\right), i=1,2, \ldots$, $n$. The total number of points in the geometry that results is $v_{1} u_{1}+v_{2} u_{2}+$ $\cdots+v_{n} u_{n}=R(k-1)+N(w+1)$. If the $\operatorname{PENT}\left(k, r_{i}, w\right), i=1,2, \ldots$, $n$, have deficiency $\left(w, 5^{+}\right)$-graphs, then by Lemma 2.4 they have pairwise disjoint opposite designs, which are clearly not affected by the blocks of the group divisible design. Therefore, again by Lemma 2.4, the deficiency graph of the constructed geometry has girth at least 5 .

\section{BLOCK SIZE 3}

By Lemma 2.1, a pentagonal geometry $\operatorname{PENT}(3, r, w)$ has $v=2 r+w+1$ points and $r(2 r+w+1) / 3$ lines. Hence $r$ is admissible if and only if $r \equiv$ 0 or $w+1(\bmod 3)$. A Steiner triple system of order $w \geq 0$ exists if and only if $w \equiv 1$ or $3(\bmod 6)$. Furthermore, $v$ is even, and for constructions using Theorem 2.5 we employ the following lemma concerning the existence of group divisible designs with block size 3 and even group sizes.

Lemma 3.1. Suppose $g \geq 1$ and $g$ is even. Then there exists a 3-GDD of type $g^{u}$ if $u \geq 3$ and $u(u-1) g \equiv 0(\bmod 3)$.

Suppose also that $m$ is even and $m \leq g(u-1)$. Then there exists a 3 -GDD of type $g^{u} m^{1}$ if $u \geq 3$ and $g^{2} u(u-1)+2 g u m \equiv 0(\bmod 3)$.

Proof. See [9] or [15, Theorem IV.4.2].

Lemma 3.2. There exists a $\operatorname{PENT}(3, r, 7)$ with connected deficiency graph of girth at least 5 for $33 \leq r \leq 149, r \equiv 0$ or $2(\bmod 3)$.

Proof. Since these geometries are not difficult to find we shall, as a reasonable alternative to presenting each one in detail, merely describe how they are obtained. Let $v=2 r+8$.

By some means create a random connected $\left(7,5^{+}\right)$-graph with $v$ vertices. The points of the geometry are the vertices of the graph. For each vertex $x$, 
construct a Steiner triple system of order 7 on the neighbours of $x$. These form the blocks of $x^{\text {opp }}$. Finally, create the remaining blocks of the geometry by hill climbing, as described in [23] or [10, Section 2.7.2]. Of course this last step is doomed if the chosen graph is not the deficiency graph of a generalized pentagonal geometry. We must therefore be prepared to restart from the beginning with a different graph if it becomes evident that the hill climbing process does not appear to be working.

We give two examples. In each case we specify $d$ followed by $d r$ numbers. To obtain the geometry, gather the numbers into triples to form a set of $d r / 3$ base blocks, $B$, which are then developed into the line set of the geometry by the mapping $x \mapsto x+d(\bmod v)$. The point set is $\{0,1, \ldots, v-1\}$ and the first $d$ sets of 7 triples of $B$ are $0^{\text {opp }}, 1^{\text {opp }}, \ldots,(d-1)^{\text {opp }}$. Further examples are given in the Appendix of this paper's preprint, which is available at

$$
\text { https://arxiv.org/abs/2104.02760. }
$$

PENT(3, 33, 7) $d=2: 5,24,49 ; 5,50,51 ; 5,59,63 ; 24,50,59 ; 24,51$, $63 ; 49,50,63 ; 49,51,59 ; 12,16,19 ; 12,24,26 ; 12,57,70 ; 16,24,57 ; 16$, 26,$70 ; 19,24,70 ; 19,26,57 ; 0,6,42 ; 0,11,53 ; 0,15,65 ; 0,17,57 ; 0,18$, $52 ; 0,19,71 ; 0,21,47 ; 0,37,43$

PENT(3, 35, 7) $d=6: 11,19,22 ; 11,58,59 ; 11,65,74 ; 19,58,65 ; 19$, 59,$74 ; 22,58,74 ; 22,59,65 ; 2,31,33 ; 2,35,39 ; 2,49,60 ; 31,35,49 ; 31$, 39,$60 ; 33,35,60 ; 33,39,49 ; 1,6,21 ; 1,29,45 ; 1,46,52 ; 6,29,46 ; 6,45$, $52 ; 21,29,52 ; 21,45,46 ; 38,40,43 ; 38,49,58 ; 38,62,70 ; 40,49,62 ; 40$, 58,$70 ; 43,49,70 ; 43,58,62 ; 15,24,27 ; 15,32,38 ; 15,45,60 ; 24,32,45$; $24,38,60 ; 27,32,60 ; 27,38,45 ; 18,23,24 ; 18,49,56 ; 18,65,72 ; 23,49$, $65 ; 23,56,72 ; 24,49,72 ; 24,56,65 ; 26,0,61 ; 18,31,19 ; 44,74,32 ; 60,42$, $46 ; 19,45,77 ; 17,20,69 ; 28,49,4 ; 45,64,3 ; 44,7,61 ; 76,11,38 ; 43,53$, $39 ; 8,18,61 ; 52,24,36 ; 33,32,11 ; 24,31,16 ; 18,27,70 ; 26,29,8 ; 68,24$, $58 ; 59,64,69 ; 29,9,58 ; 24,51,26 ; 63,13,49 ; 4,6,23 ; 31,9,53 ; 0,10,75$; $0,35,37 ; 2,16,41 ; 3,41,53$

Theorem 3.1. There exists a $\operatorname{PENT}(3, r, 7)$ with deficiency graph of girth at least 5 if and only if $r \geq 21$ and $r \equiv 0$ or $2(\bmod 3)$, except possibly for $r \in\{23,24,26,27,29,30,32\}$.

Proof. The smallest $(7,5)$-graph is the 50-vertex Hoffman-Singleton graph, which yields a $\operatorname{PENT}(3,21,7)$ by Theorem 2.3 . Therefore, by Lemma 3.2, we need to consider only admissible $r \geq 150$.

Let $S_{7}=\{s: 33 \leq s \leq 71, s \equiv 0$ or $2(\bmod 3)\}$ and observe that the elements of $S_{7}$ cover the admissible residues modulo 39. By Lemma 3.2, there exists a $\operatorname{PENT}(3, s, 7)$ for $s \in S_{7}$.

For each $s \in S_{7}$, take $u$ copies of a $\operatorname{PENT}(3,35,7)$, which has 78 points, and one copy of a $\operatorname{PENT}(3, s, 7)$, which has $2 s+8$ points. By Lemma 3.1, since $2 s+8 \leq 78 \cdot 2$, there exists a 3 -GDD of type $78^{u}(2 s+8)^{1}$ for $u=3,4$, ... Now use Theorem 2.5 to construct a $\operatorname{PENT}(3,39 u+s, 7)$ for $u \geq 3$.

Hence there exists a $\operatorname{PENT}(3, r, 7)$ for admissible $r \geq 150$. 
Lemma 3.3. There exists a $\operatorname{PENT}(3, r, 9)$ with connected deficiency graph of girth at least 5 for $57 \leq r \leq 244, r \equiv 0$ or $1(\bmod 3)$.

Proof. For each of the stated values of $r$, create a random connected $\left(9,5^{+}\right)$graph with $2 r+10$ vertices. On the neighbours of each point $x$, construct a Steiner triple system of order 9 to form the blocks of $x^{\text {opp }}$. Create the remaining blocks of the geometry by hill climbing. The process is readily automated. Some examples appear in the paper's ArXiv preprint.

Theorem 3.2. There exists a $\mathrm{PENT}(3, r, 9)$ with deficiency graph of girth at least 5 if $r \equiv 0$ or $1(\bmod 3)$ and $r \geq 57$.

Proof. The main argument is similar to that of Theorem 3.1.

Let $S_{9}=\{s: 57 \leq s \leq 118, s \equiv 0$ or $1(\bmod 3)\}$ and observe that this set covers the admissible residues modulo 63. By Lemma 3.3, there exists a $\operatorname{PENT}(3, s, 9)$ for $s \in S_{9}$, and it suffices to address only $r \geq 246$.

For each $s \in S_{9}$, take $u$ copies of a $\operatorname{PENT}(3,58,9)$, which has 126 points, and one copy of a $\operatorname{PENT}(3, s, 9)$. By Lemma 3.1 , since $2 s+10 \leq 126 \cdot 2$, there exists a 3 -GDD of type $126^{u}(2 s+10)^{1}$ for $u=3,4, \ldots$ Now use Theorem 2.5 to construct a $\operatorname{PENT}(3,63 u+s, 9)$ for $u \geq 3$.

Hence there exists a $\operatorname{PENT}(3, r, 9)$ for admissible $r \geq 3 \cdot 63+57=246$.

It is clear that one can continue in a similar manner until one's computers run out of steam. With the availability of sufficient resources, 'starter systems' can be obtained to prove similar theorems for $w>9$ that are valid as orders of Steiner triple systems. We continue by dealing briefly with $w \in$ $\{13,15,19,21,25,27,31,33\}$.

Lemma 3.4. There exists a $\operatorname{PENT}(3, r, w)$ with connected deficiency graph of girth at least 5 for

$$
\begin{aligned}
(r, w) \in\{ & (168,13),(170,13),(176,13), \\
& (249,15),(253,15),(259,15), \\
& (483,19),(485,19),(491,19), \\
& (654,21),(658,21),(664,21), \\
& (1212,25),(1214,25),(1220,25), \\
& (1389,27),(1393,27),(1399,27), \\
& (2133,31),(2135,31),(2141,31), \\
& (2550,33),(2554,33),(2560,33)\} .
\end{aligned}
$$

Proof. These are created as in Lemma 3.2 from random connected $\left(w, 5^{+}\right)$graphs with $v=2 r+w+1$ vertices. Some examples appear in the paper's ArXiv preprint. Alternatively, you may email the first author for the entire collection (including those of Lemmas 3.2 and 3.3). We restrict $r$ to values where the number of points in the corresponding $\operatorname{PENT}(3, r, w)$ is congruent to 2 modulo 4 . With this constraint we are able to exploit an automorphism of the form $x \mapsto x+2(\bmod v)$ or $x \mapsto x+6(\bmod v)$. 
Theorem 3.3. Let $w_{0}=1$ or 3 . Let $w, r_{0}, r_{1}$ and $r_{2}$ be positive integers satisfying $w \geq 7, w \equiv w_{0}(\bmod 6), r_{0} \equiv 0(\bmod 3), 2 r_{0}+w+1 \equiv 2(\bmod 4)$, $r_{1}=r_{0}+w_{0}+1$, and $r_{2}=r_{1}+6$.

Suppose that for $i \in\{0,1,2\}$, there exist a generalized pentagonal geometry $\operatorname{PENT}\left(3, r_{i}, w\right)$, and let $g$ be the minimum of the girths of their deficiency graphs. Then for all sufficiently large $r \equiv 0$ or $w+1(\bmod 3)$, there exist a generalized pentagonal geometry $\operatorname{PENT}(3, r, w)$ with deficiency graph of girth at least $g$.

Proof. Let $v_{i}=2 r_{i}+w+1, i=0,1,2$, and note that $v_{1} \equiv v_{2} \equiv 6(\bmod 12)$. Let $r_{*} \in\left\{r_{0}, r_{1}\right\}$ and $v_{*}=2 r_{*}+w+1$.

Apply Theorem 2.5 twice, first with a $\operatorname{PENT}\left(3, r_{*}, w\right), t$ copies of a $\operatorname{PENT}\left(3, r_{1}, w\right)$ and a 3 -GDD of type $v_{1}^{t} v_{*}^{1}$ to obtain a

$$
\operatorname{PENT}\left(3, v_{1} t / 2+r_{*}, w\right) \text { for } t \geq 3,
$$

and then with $u$ copies of a $\operatorname{PENT}\left(3, r_{2}, w\right)$ and a 3 -GDD of type $v_{2}^{u}\left(v_{1} t+v_{*}\right)^{1}$ to obtain a

$$
\operatorname{PENT}\left(3, v_{2} u / 2+v_{1} t / 2+r_{*}, w\right) \text { for } u \geq t+2, t \geq 3 .
$$

To confirm the existence of the two 3-GDDs by Lemma 3.1, we have $v_{1} \equiv$ $v_{2} \equiv 0(\bmod 6), v_{*} \equiv 0(\bmod 2), v_{*} \leq v_{1} \leq v_{1}(t-1)$ and $v_{1} t+v_{*} \leq$ $v_{2}(t+1) \leq v_{2}(u-1)$.

Observe that in (5) the greatest common divisor of the coefficients of $u$ and $t$ is 3 , and that the two options for $r_{*}$ belong to the two admissible residue classes modulo 3 . Hence, by an argument involving the Chinese Remainder Theorem similar to that in the proof of Theorem 4.1, below, the range of $v_{2} u / 2+v_{1} t / 2+r_{*}$ in (5) covers all sufficiently large admissible values.

It is clear that the process cannot introduce a cycle of fewer than $g$ vertices into the deficiency graph of the constructed geometry.

Theorem 3.4. For each $w \in\{13,15,19,21,25,27,31,33\}$, there exist pentagonal geometries $\operatorname{PENT}(3, r, w)$ with deficiency graph of girth at least 5 for all sufficiently large $r \equiv 0$ or $w+1(\bmod 3)$.

Proof. This follows from Lemma 3.4 and Theorem 3.3.

We conclude Section 3 by constructing a $\operatorname{PENT}(3,51,7)$ with connected deficiency graph of girth 4 . In contrast with the geometries that we have hitherto considered in this section, there exist distinct $x, y$ where the block sets of $x^{\text {opp }}$ and $y^{\text {opp }}$ have a common block. However, the point sets of $x^{\text {opp }}$ and $y^{\text {opp }}$ never coincide for $x \neq y$ and therefore the geometry does not contain an opposite design pair.

Let the point set of the $\operatorname{PENT}(3,51,7)$ be $\{0,1, \ldots, 109\}$. Create the edges of a $(7,4)$-graph $D$ on 110 vertices from the set

$$
\{\{0,1\},\{0,7\},\{0,23\},\{0,35\},\{0,39\},\{0,59\},\{0,75\}\}
$$


developed by the mapping $x \mapsto x+2(\bmod 110)$. For a vertex $x$ of $D$, let $N(x)=\{z: z \sim x\}$, the set of neighbours of $x$. The graph $D$ has the following properties (Figure 2).

(i) For any vertex $x$, we have $|N(x)|=7$.

(ii) For any vertices $x, y, x \neq y$, we have $|N(x) \cap N(y)| \in\{0,1,3\}$.

(iii) Two neighbourhood intersections of size 3 never meet in precisely two points. This is necessary for consistently overlaying the neighbourhoods with STS(7)s.

(iv) For each vertex $x$ of $D$, there is a set $T(x)=\left\{T_{1}, T_{2}, T_{3}\right\}$ of three distinct triples, $T_{1}, T_{2}, T_{3}$, and 6 distinct vertices $\left\{y_{1}, z_{1}, y_{2}, z_{2}, y_{3}, z_{3}\right\}$, also distinct from $N(x) \cup\{x\}$, such that $\left|T_{1} \cup T_{2} \cup T_{3}\right|=6$ and

$$
T_{i}=N(x) \cap N\left(y_{i}\right)=N(x) \cap N\left(z_{i}\right), i=1,2,3 .
$$

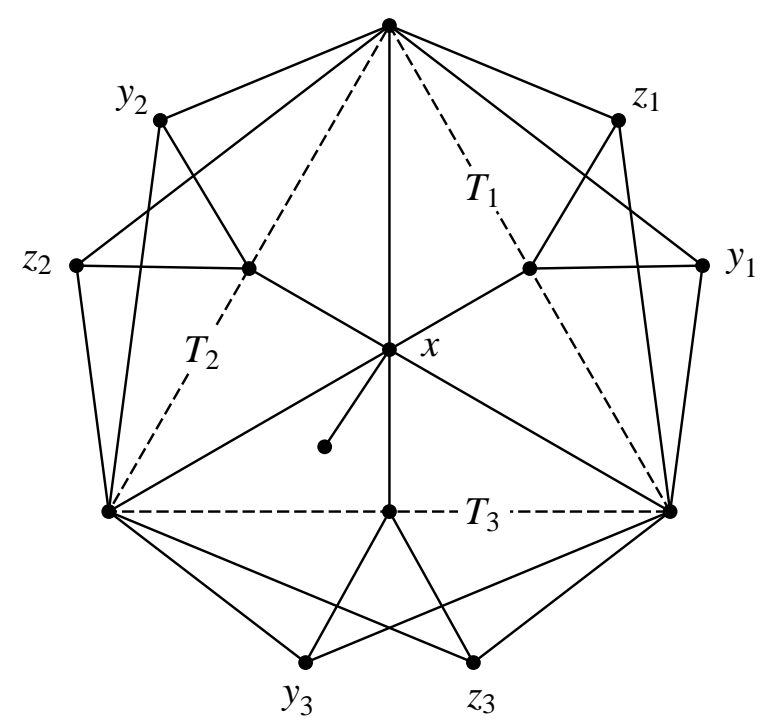

Figure 2. PENT(3,51,7): neighbours of $x$

For each point $x$, the point set of $x^{\mathrm{opp}}$ is $N(x)$ and to construct the block set of $x^{\text {opp }}$ we extend $T(x)$ to an $\operatorname{STS}(7)$ on $N(x)$. After discarding duplicates the $110 \cdot 5$ distinct lines so constructed form the 550 lines that belong to opposite designs. The remaining 1320 lines are found by hill climbing. To obtain the 1870 lines of the geometry, partition the 102 numbers specified below into 34 triples. Then develop the triples with the mapping $x \mapsto$ $x+2(\bmod 110)$.

PENT(3, 51, 7): 0, 36, 52; 0, 72, 104; 0, 76, 88; 1, 7, 39; 1, 23, 35; 1, 59, $75 ; 7,35,75 ; 35,39,59 ; 36,76,104 ; 52,72,76 ; 0,2,49 ; 0,3,57 ; 0,5,54$; $0,8,25 ; 0,9,11 ; 0,10,97 ; 0,13,96 ; 0,15,107 ; 0,18,95 ; 0,19,60 ; 0,21$, $51 ; 0,26,67 ; 0,29,73 ; 0,30,101 ; 0,31,93 ; 0,33,83 ; 0,37,44 ; 0,43,48$; $0,45,64 ; 0,53,79 ; 0,55,65 ; 0,63,109 ; 0,81,89 ; 0,85,99$ 


\section{BLOCK SIZE 4}

By Lemma 2.1, a pentagonal geometry $\operatorname{PENT}(4, r, w)$ has $3 r+w+1$ points and $r(3 r+w+1) / 4$ lines. Furthermore, a Steiner system $S(2,4, w)$ with positive $w$ exists if and only if $w \equiv 1$ or $4(\bmod 12)$. Therefore we require $r \equiv 0$ or $w+1(\bmod 4)$. In contrast to the case $k=3$, examples of $\operatorname{PENT}(k, r, w)$ with $w>k>3$ and pairwise disjoint opposite designs appear to be quite difficult to find. The fourteen items listed in Lemma 4.1, below, are the only such geometries known to the authors.

Lemma 4.1. There exists a $\operatorname{PENT}(4, r, 13)$ with connected deficiency graph of girth 5 if $r \in\{112,116,120,124,128,132,136,140,144,148,152,156$, $160,164\}$.

Proof. In each case we specify $2 r$ numbers. To obtain the geometry, gather the numbers into quadruples to form a set of $r / 2$ base blocks, $B$, which are developed into the line set of the geometry by the mapping $x \mapsto x+$ $2(\bmod 3 r+14)$. The first two sets of 13 quadruples of $B$ are $0^{\text {opp }}$ and $1^{\text {opp }}$. The opposite designs are projective planes of order 3 . The deficiency graphs have girth 5 .

PENT(4, 112, 13): 21, 41, 49, 297; 21, 140, 210, 319; 21, 149, 293, 335; $21,163,287,337 ; 41,140,293,337 ; 41,149,287,319 ; 41,163,210,335 ; 49$, $140,287,335 ; 49,149,210,337 ; 49,163,293,319 ; 140,149,163,297 ; 210$, 287, 293, 297; 297, 319, 335, 337; 14, 16, 32, 293; 14, 54, 64, 302; 14, 58, 202,$310 ; 14,59,188,330 ; 16,54,202,330 ; 16,58,188,302 ; 16,59,64,310$; $32,54,188,310 ; 32,58,64,330 ; 32,59,202,302 ; 54,58,59,293 ; 64,188$, 202, 293; 293, 302, 310, 330; 110, 249, 50, 261; 206, 137, 183, 48; 247, 154, 277,$34 ; 132,347,323,292 ; 34,58,37,189 ; 167,71,106,340 ; 196,346,269$, $11 ; 0,7,46,291 ; 0,13,133,136 ; 0,11,137,264 ; 0,17,198,217 ; 0,25,110$, $185 ; 0,68,209,269 ; 0,51,119,126 ; 0,58,187,305 ; 0,63,82,323 ; 0,35,76$, $145 ; 0,33,88,307 ; 0,47,205,254 ; 0,115,166,325 ; 0,29,117,284 ; 0,92$, 203,$349 ; 0,39,118,175 ; 0,113,146,267 ; 0,90,193,263 ; 0,53,154,321$; $0,85,171,182 ; 0,65,132,233 ; 0,12,71,237 ; 0,30,67,285$

PENT(4, 116, 13): 9, 20, 39, 277; 9, 55, 141, 311; 9, 63, 267, 329; 9, 77, 147,$342 ; 20,55,267,342 ; 20,63,147,311 ; 20,77,141,329 ; 39,55,147$, $329 ; 39,63,141,342 ; 39,77,267,311 ; 55,63,77,277 ; 141,147,267,277$; $277,311,329,342 ; 34,52,57,307 ; 34,86,222$, 308; 34, 96, 300, 324; 34, $216,286,354 ; 52,86,300,354 ; 52,96,286,308 ; 52,216,222,324 ; 57,86$, 286,$324 ; 57,96,222,354 ; 57,216,300,308 ; 86,96,216,307 ; 222,286,300$, 307 ; 307, 308, 324, 354; 217, 15, 206, 64; 84, 65, 236, 39; 159, 303, 66, 34; $206,105,304,221 ; 148,79,197,264 ; 268,309,305,82 ; 3,294,83,220$; 81, $8,237,192 ; 6,107,268,9 ; 206,341,38,254 ; 159,314,326,101 ; 350,231$, 44, 307; 281, 159, 127, 168; 0, 1, 28, 281; 0, 61, 66, 239; 0, 58, 175, 341; 0, $80,189,289 ; 0,47,144,359 ; 0,112,235,271 ; 0,31,199,312 ; 0,4,217,245$; $0,33,53,326 ; 0,67,219,244 ; 0,99,160,275 ; 0,17,233,240 ; 0,51,154$, $347 ; 0,25,27,82 ; 0,129,169,206 ; 0,13,76,155 ; 0,131,143,166 ; 0,26$, 183,$231 ; 0,2,107,181$ 
PENT(4, 120, 13): 11, 18, 31, 309; 11, 97, 263, 325; 11, 109, 295, 356; 11, 261, 269, 363; 18, 97, 295, 363; 18, 109, 269, 325; 18, 261, 263, 356; 31, 97, 269, 356; 31, 109, 263, 363; 31, 261, 295, 325; 97, 109, 261, 309; 263, 269, 295,$309 ; 309,325,356,363 ; 12,50,66,278 ; 12,71,112,305 ; 12,80,266$, $344 ; 12,106,114,364 ; 50,71,266,364 ; 50,80,114,305 ; 50,106,112,344$; $66,71,114,344 ; 66,80,112,364 ; 66,106,266,305 ; 71,80,106,278 ; 112$, 114, 266, 278; 278, 305, 344, 364; 236, 99, 308, 47; 55, 46, 185, 288; 162, 41, 371,$246 ; 108,39,339,314 ; 290,145,323,262 ; 60,295,152,101 ; 247,192$, 189,$58 ; 310,300,367,185 ; 160,83,343,340 ; 132,355,4,115 ; 32,210,155$, $348 ; 9,116,235,220 ; 0,1,19,322 ; 0,4,107,304 ; 0,17,145,170 ; 0,23,161$, $262 ; 0,35,45,324 ; 0,65,137,321 ; 0,24,171,341 ; 0,44,133,217 ; 0,51$, 192,$275 ; 0,121,140,289 ; 0,43,175,190 ; 0,75,201,292 ; 0,63,219,224$; $0,37,87,256 ; 0,47,151,226 ; 0,73,77,244 ; 0,126,285,373 ; 0,114,301$, $329 ; 0,105,197,268 ; 0,88,241,353 ; 0,69,337,361 ; 0,93,129,156$

PENT(4, 124, 13): 19, 37, 82, 285; 19, 151, 211, 304; 19, 205, 247, 311; $19,207,239,355 ; 37,151,247,355 ; 37,205,239,304 ; 37,207,211,311 ; 82$, $151,239,311 ; 82,205,211,355 ; 82,207,247,304 ; 151,205,207,285$; 211 , $239,247,285 ; 285,304,311,355 ; 32,76,99,236 ; 32,102,176,289 ; 32,140$, 182,$350 ; 32,148,180,368 ; 76,102,182,368 ; 76,140,180,289 ; 76,148$, 176,$350 ; 99,102,180,350 ; 99,140,176,368 ; 99,148,182,289 ; 102,140$, 148,$236 ; 176,180,182,236 ; 236,289,350,368 ; 230,363,94,79 ; 258,335$, 95,$306 ; 252,63,80,93 ; 30,249,25,54 ; 30,281,223,14 ; 92,336,5,16 ; 252$, $209,3,62 ; 79,38,13,3 ; 380,209,78,69 ; 191,312,65,214 ; 374,168,279$, $23 ; 15,237,308,12 ; 145,332,167,214 ; 260,233,2,1 ; 61,365,326,164$; $293,214,204,231 ; 0,1,143,373 ; 0,11,279,300 ; 0,12,179,331 ; 0,47,95$, $264 ; 0,45,52,178 ; 0,65,156,243 ; 0,22,281,297 ; 0,5,57,130 ; 0,15,277$, $328 ; 0,49,246,349 ; 0,14,173,185 ; 0,30,63,191 ; 0,43,153,284 ; 0,59$, 181,$353 ; 0,9,99,240 ; 0,25,110,234 ; 0,21,271,324 ; 0,71,91,294 ; 0,31$, 115,$154 ; 0,20,81,105$

PENT(4, 128, 13): 341, 43, 86, 117; 341, 71, 312, 349; 341, 351, 381, $165 ; 341,97,121,55 ; 43,71,381,55 ; 43,351,121,349 ; 43,97,312,165 ; 86$, $71,121,165 ; 86,351,312,55 ; 86,97,381,349 ; 71,351,97,117 ; 312,121$, 381,$117 ; 117,349,165,55 ; 302,18,58,234 ; 302,50,282,328 ; 302$, 344, 356,$278 ; 302,151,249,48 ; 18,50,356,48 ; 18,344,249,328 ; 18,151,282$, 278 ; 58, 50, 249, 278; 58, 344, 282, 48; 58, 151, 356, 328; 50, 344, 151, 234; $282,249,356,234 ; 234,328,278,48 ; 55,345,156,118 ; 291,202,286,14$; $251,178,55,195 ; 184,97,364,308 ; 291,131,136,130 ; 79,220,143,144$; $287,176,274,15 ; 207,166,230,131 ; 299,148,310,357 ; 94,57,265,116$; $192,19,269,174 ; 23,180,286,1 ; 140,3,239,10 ; 293,151,178,260 ; 112$, $203,51,254 ; 0,3,248,395 ; 0,9,197,238 ; 0,14,204,249 ; 0,7,233,318$; $0,21,59,140 ; 0,19,132,213 ; 0,108,245,331 ; 0,65,158,283 ; 0,173,192$, $373 ; 0,23,242,385 ; 0,107,159,239 ; 0,83,200,323 ; 0,53,177,259 ; 0,105$, 175,$196 ; 0,63,250,325 ; 0,61,329,343 ; 0,58,167,185 ; 0,52,269,353 ; 0$, $27,116,152 ; 0,57,96,163 ; 0,25,136,389 ; 0,34,85,327 ; 0,49,145,328$ 
PENT(4, 132, 13): 5, 23, 75, 289; 5, 89, 187, 377; 5, 129, 278, 399; 5, $132,247,403 ; 23,89,278,403 ; 23,129,247,377 ; 23,132,187,399 ; 75,89$, 247,$399 ; 75,129,187,403 ; 75,132,278,377 ; 89,129,132,289 ; 187,247$, 278,$289 ; 289,377,399,403 ; 8,12,34,322 ; 8,105,224,336 ; 8,122,307$, $388 ; 8,164,282,406 ; 12,105,307,406 ; 12,122,282,336 ; 12,164,224,388$; $34,105,282,388 ; 34,122,224,406 ; 34,164,307,336 ; 105,122,164,322$; $224,282,307,322 ; 322,336,388,406 ; 77,262,114,218$; 132, 298, 345, 111; $80,401,340,183 ; 154,64,0,81 ; 340,166,119,375 ; 69,392,347,120 ; 400$, $233,29,380 ; 38,249,313,323 ; 55,229,264,188 ; 63,76,68,113 ; 27,291$, 54,$132 ; 74,392,221,159 ; 397,182,40,259 ; 170,15,17,277 ; 84,225,290$, $156 ; 93,86,262,312 ; 0,1,29,408 ; 0,10,63,205 ; 0,6,15,181 ; 0,19,27$, $94 ; 0,13,32,197 ; 0,21,113,374 ; 0,28,95,173 ; 0,24,341,347 ; 0,133,153$, $230 ; 0,111,135,218 ; 0,46,119,151 ; 0,116,279,315 ; 0,101,169,217 ; 0$, $43,123,362 ; 0,33,161,240 ; 0,62,297,369 ; 0,231,325,401 ; 0,249,293$, 339 ; 0, 120, 251, 385; 0, 49, 281, 342; 0, 65, 188, 355; 0, 139, 178, 361; 0, 79, 80,$309 ; 0,51,74,202$

PENT(4, 136, 13): 343, 209, 397, 416; 343, 325, 1, 301; 343, 287, 119, 6; 343, 389, 49, 51; 209, 325, 119, 51; 209, 287, 49, 301; 209, 389, 1, 6; 397, $325,49,6 ; 397,287,1,51 ; 397,389,119,301 ; 325,287,389,416 ; 1,49,119$, 416 ; 416, 301, 6, 51; 304, 98, 374, 413; 304, 214, 0, 136; 304, 122, 80, 11; $304,372,26,34 ; 98,214,80,34 ; 98,122,26,136 ; 98,372,0,11 ; 374,214$, 26,$11 ; 374,122,0,34 ; 374,372,80,136 ; 214,122,372,413 ; 0,26,80,413$; $413,136,11,34 ; 334,59,268,339 ; 371,50,228,145 ; 56,76,339,213 ; 148$, $89,288,321 ; 180,220,297,30 ; 295,168,191,131 ; 207,102,132,256 ; 28$, $312,337,65 ; 366,29,240,51 ; 24,130,295,229 ; 52,233,23,166 ; 87,145$, 240,$292 ; 365,138,334,57 ; 80,390,79,335 ; 400,215,129,206 ; 391,408$, 101,$162 ; 186,369,306,123 ; 234,5,296,202 ; 128,5,340,57 ; 293,44,289$, 333 ; 0, 3, 290, 305; 0, 10, 27, 89; 0, 29, 200, 204; 0, 19, 60, 318; 0, 13, 187, $280 ; 0,22,265,377 ; 0,86,317,347 ; 0,103,156,323 ; 0,73,260,401 ; 0,16$, 149,$185 ; 0,58,179,371 ; 0,21,221,415 ; 0,163,195,303 ; 0,69,81,314 ; 0$, $161,174,365 ; 0,257,273,411 ; 0,36,83,207$; 0, 28, 93, 230; 0, 35, 256, 357; $0,91,97,253 ; 0,53,159,322 ; 0,44,241,419$

PENT(4, 140, 13): 8, 9, 109, 379; 8, 159, 295, 383; 8, 203, 327, 401; 8, 213, 321, 426; 9, 159, 327, 426; 9, 203, 321, 383; 9, 213, 295, 401; 109, 159, 321,$401 ; 109,203,295,426$; 109, 213, 327, 383; 159, 203, 213, 379; 295, 321, 327,$379 ; 379,383,401,426 ; 34,52,56,276 ; 34,79,140,326 ; 34,108,232$, $357 ; 34,114,222,426 ; 52,79,232,426 ; 52,108,222,326 ; 52,114,140,357$; $56,79,222,357 ; 56,108,140,426 ; 56,114,232,326 ; 79,108,114,276 ; 140$, $222,232,276$; 276, 326, 357, 426; 388, 155, 59, 52; 364, 352, 351, 20; 268, $192,185,420 ; 350,385,70,118 ; 140,142,75,375 ; 281,382,301,421 ; 203$, $398,57,142 ; 389,403,312,8 ; 153,304,170,1 ; 239,404,366,43 ; 14,240$, 369,$61 ; 260,3,79,403 ; 421,424,58,242 ; 62,201,48,333 ; 272,257,160$, $85 ; 293,338,314,17 ; 244,388,147,0 ; 101,34,191,64 ; 126,267,405,221$; $172,94,345,357 ; 150,339,369,350 ; 0,3,5,96 ; 0,13,40,115 ; 0,11,172$, 347 ; 0, 15, 34, 197; 0, 57, 276, 361; 0, 25, 63, 120; 0, 59, 171, 348; 0, 43, 66, 
$275 ; 0,41,72,241 ; 0,128,261,309 ; 0,21,149,165 ; 0,28,183,324 ; 0,71$, 79,$107 ; 0,83,289,294 ; 0,29,53,398 ; 0,20,119,345 ; 0,69,146,278 ; 0$, 49, 121, 238; 0, 126, 385, 425; 0, 70, 231, 297; 0, 51, 174, 229; 0, 123, 191, $277 ; 0,33,207,397$

PENT(4, 144, 13): 73, 93, 99, 267; 73, 101, 145, 336; 73, 110, 225, 431; 73, 131, 155, 443; 93, 101, 225, 443; 93, 110, 155, 336; 93, 131, 145, 431; 99, $101,155,431 ; 99,110,145,443 ; 99,131,225,336 ; 101,110,131,267 ; 145$, $155,225,267 ; 267,336,431,443 ; 4,16,129,346 ; 4,180,302,348 ; 4,222$, 319,$354 ; 4,292,316,374 ; 16,180,319,374 ; 16,222,316,348 ; 16,292$, 302, $354 ; 129,180,316,354 ; 129,222,302,374 ; 129,292,319,348 ; 180,222$, 292,$346 ; 302,316,319,346 ; 346,348,354,374 ; 382,283,296,86 ; 161,7$, 251,$168 ; 178,307,160,239 ; 222,218,158,29 ; 135,72,87,364 ; 213,416$, 333,158 ; 220, 297, 48, 67; 108, 18, 141, 67; 129, 311, 116, 312; 11, 54, 372, 29 ; 160, 238, 99, 373; 339, 402, 335, 275; 219, 428, 250, 272; 365, 3, 42, 308; $354,281,120,189 ; 321,59,148,378 ; 134,27,254,293 ; 103,357,206,317$; 7, 66, 206, 257; 34, 388, 1, 338; 123, 321, 204, 37; 84, 48, 409, 132; 357, 66, 320,$171 ; 198,164,412,317 ; 0,1,242,372 ; 0,5,106,341 ; 0,16,41,179 ; 0$, $98,269,399 ; 0,7,246,313 ; 0,43,66,289 ; 0,23,89,224 ; 0,53,87,238 ; 0$, $91,182,359 ; 0,68,299,397 ; 0,71,121,186 ; 0,167,189,425 ; 0,9,118,305$; $0,199,215,441 ; 0,29,251,369 ; 0,11,47,308 ; 0,40,184,327 ; 0,100,233$, $375 ; 0,31,239,284 ; 0,65,202,367 ; 0,81,181,256 ; 0,137,349,427$

PENT(4, 148, 13): 79, 41, 147, 259; 79, 96, 49, 283; 79, 111, 362, 277; $79,417,415,427 ; 41,96,362,427 ; 41,111,415,283 ; 41,417,49,277 ; 147$, $96,415,277 ; 147,111,49,427 ; 147,417,362,283 ; 96,111,417,259 ; 49$, $415,362,259 ; 259,283,277,427 ; 44,32,182,200 ; 44,101,42,348 ; 44,176$, 359,312 ; 44, 410, 380, 418; 32, 101, 359, 418; 32, 176, 380, 348; 32, 410, 42, 312 ; 182, 101, 380, 312; 182, 176, 42, 418; 182, 410, 359, 348; 101, 176, 410, $200 ; 42,380,359,200 ; 200,348,312,418 ; 3,322,198,187 ; 396,52,129$, 223 ; 111, 302, 229, 396; 244, 284, 265, 156; 256, 242, 15, 93; 330, 5, 54, 415; 194, 217, 340, 345; 385, 352, 410, 394; 435, 183, 448, 391; 23, 180, 374, 111; 431, 33, 317, 226; 44, 18, 426, 317; 387, 146, 241, 227; 404, 296, 413, 178; 418, 199, 113, 314; 126, 145, 91, 78; 119, 284, 123, 306; 446, 276, 237, 301; $433,2,169,68 ; 102,215,189,139 ; 285,440,338,227 ; 196,119,385,224$; 176, 130, 251, 428; 0, 1, 414, 443; 0, 3, 43, 372; 0, 4, 212, 219; 0, 17, 20, 196; 0, 27, 342, 457; 0, 31, 201, 406; 0, 54, 209, 421; 0, 71, 78, 333; 0, 99, 296, 453; 0, 197, 305, 429; 0, 103, 174, 397; 0, 39, 61, 294; 0, 123, 169, 256; 0, 93, 126, 200; 0, 63, 165, 394; 0, 35, 237, 358; 0, 97, 341, 393; 0, 131, 214, 413; 0, $184,369,435 ; 0,105,125,221 ; 0,56,289,363 ; 0,60,287,329 ; 0,213,339$, $395 ; 0,73,186,220 ; 0,119,357,391$

PENT(4, 152, 13): 26, 105, 125, 365; 26, 127, 259, 389; 26, 165, 307, 419; 26, 227, 293, 444; 105, 127, 307, 444; 105, 165, 293, 389; 105, 227, 259, 419; 125, 127, 293, 419; 125, 165, 259, 444; 125, 227, 307, 389; 127, 165, 227, $365 ; 259,293,307,365 ; 365,389,419,444 ; 52,82,106,344 ; 52,111,212$, 346 ; 52, 164, 306, 361; 52, 178, 244, 366; 82, 111, 306, 366; 82, 164, 244, 346 ; 82, 178, 212, 361; 106, 111, 244, 361; 106, 164, 212, 366; 106, 178, 306, 
$346 ; 111,164,178,344 ; 212,244,306,344 ; 344,346,361,366 ; 38,20,251$, 225 ; 383, 270, 341, 406; 426, 208, 227, 318; 135, 153, 198, 128; 134, 447, 411, $362 ; 404,8,405,417 ; 398,352,208,315 ; 450,361,120,143 ; 256,417,272$, $152 ; 12,469,15,365 ; 251,58,284,280 ; 249,199,20,76 ; 187,230,339,258$; $70,394,273,1 ; 290,149,155,380 ; 23,159,324,150 ; 255,456,180,27 ; 153$, $100,24,349 ; 70,184,127,331 ; 372,257,205,72 ; 231,204,319,20 ; 80,88$, 44, 300; 444, 347, 455, 61; 122, 443, 128, 19; 223, 59, 178, 188; 133, 72, 431, $394 ; 0,21,31,428 ; 0,33,118,157 ; 0,43,47,420 ; 0,51,346,429 ; 0,65,92$, $181 ; 0,12,171,318 ; 0,98,371,455 ; 0,95,274,395 ; 0,64,167,311 ; 0,84$, 327,$467 ; 0,137,172,459 ; 0,69,88,283 ; 0,116,251,463 ; 0,331,421,449$; $0,199,207,263 ; 0,78,301,375 ; 0,143,221,289 ; 0,225,295,469 ; 0,67$, 111,$423 ; 0,86,177,236 ; 0,141,158,377 ; 0,155,345,431 ; 0,68,257,266$; $0,119,217,439$

PENT(4, 156, 13): 115, 155, 170, 337; 115, 193, 309, 351; 115, 219, 319, $411 ; 115,275,312$, 459; 155, 193, 319, 459; 155, 219, 312, 351; 155, 275, 309, $411 ; 170,193,312$, 411; 170, 219, 309, 459; 170, 275, 319, 351; 193, 219, 275, 337 ; 309, 312, 319, 337; 337, 351, 411, 459; 24, 72, 132, 277; 24, 146, 207, $290 ; 24,164,264,328 ; 24,174,208,368 ; 72,146,264,368 ; 72,164,208,290$; 72, 174, 207, 328; 132, 146, 208, 328; 132, 164, 207, 368; 132, 174, 264, 290; 146, 164, 174, 277; 207, 208, 264, 277; 277, 290, 328, 368; 192, 120, 431, 229; $351,228,174,380 ; 158,481,268,44 ; 369,135,442,28 ; 21,293,160,405$; $52,326,406,136 ; 470,459,296,247 ; 37,287,76,13 ; 61,213,378,94 ; 95$, 206, 429, 6; 467, 242, 305, 479; 105, 476, 140, 460; 473, 119, 380, 432; 415, $328,230,409 ; 46,237,278,34 ; 2,159,301,454 ; 146,439,126,343 ; 133,80$, 205,$371 ; 382,287,477,468 ; 410,117,34,81 ; 173,298,118,320 ; 327,226$, 176, 97; 65, 168, 292, 433; 254, 55, 464, 3; 241, 68, 302, 329; 386, 401, 132, $405 ; 256,335,333,422 ; 0,1,6,17 ; 0,5,35,220 ; 0,24,172,257 ; 0,4,134$, $199 ; 0,29,51,230 ; 0,3,201,388 ; 0,36,271,401 ; 0,31,251,345 ; 0,2,279$, 333 ; 0, 88, 209, 463; 0, 96, 383, 451; 0, 59, 143, 168; 0, 161, 227, 307; 0, 71, 247,$381 ; 0,243,263,349 ; 0,67,369,412 ; 0,57,66,315 ; 0,159,176,347$; $0,46,405,413 ; 0,58,385,435 ; 0,45,188,417 ; 0,8,215,415 ; 0,129,397$, $455 ; 0,91,112,268 ; 0,117,429,475$

PENT(4, 160, 13): 39, 87, 137, 309; 39, 139, 247, 315; 39, 221, 293, 323; $39,238,256,489 ; 87,139,293,489 ; 87,221,256,315 ; 87,238,247,323$; 137, 139, 256, 323; 137, 221, 247, 489; 137, 238, 293, 315; 139, 221, 238, 309; $247,256,293,309 ; 309,315,323,489 ; 6,153,172,356 ; 6,180,248,358 ; 6$, $186,343,408 ; 6,202,274,456 ; 153,180,343,456 ; 153,186,274,358 ; 153$, 202, 248, 408; 172, 180, 274, 408; 172, 186, 248, 456; 172, 202, 343, 358; 180, $186,202,356 ; 248,274,343,356 ; 356,358,408,456 ; 260,163,223,384 ; 151$, $466,55,346 ; 337,172,149,420 ; 347,334,283,492 ; 391,311,44,187 ; 264$, $1,441,452 ; 161,329,438,58 ; 415,439,50,264 ; 251,307,5,218 ; 139,34$, 35,$124 ; 209,79,4,205 ; 466,269,424,179 ; 385,443,432,463 ; 28,51,310$, $183 ; 240,402$, 442, 483; 319, 107, 16, 210; 365, 393, 136, 300; 206, 322, 263, $43 ; 386,248,295,260 ; 468,327,489,35 ; 228,113,103,491 ; 206,436,225$, $286 ; 26,439,492,327 ; 346,269,154,404 ; 4,413,100,204 ; 185,122,52,16$; 
298, 362, 493, 308; 38, 157, 355, 373; 0, 3, 24, 123; 0, 4, 220, 431; 0, 5, 364, $371 ; 0,27,56,101 ; 0,20,187,327 ; 0,29,140,262 ; 0,60,241,279 ; 0,34$, 345,$416 ; 0,49,74,425 ; 0,17,148,361 ; 0,32,159,408 ; 0,97,304,453 ; 0$, $73,305,419 ; 0,125,161,204 ; 0,117,129,358$; 0, 51, 173, 348; 0, 227, 421, $491 ; 0,183,225,463 ; 0,199,357,493 ; 0,107,342,455 ; 0,79,111,145 ; 0$, $153,198,387 ; 0,66,321,407 ; 0,38,275,439 ; 0,121,128,260 ; 0,25,269$, 326

PENT(4, 164, 13): 67, 142, 213, 364; 67, 221, 271, 433; 67, 227, 309, 437; 67, 269, 281, 459; 142, 221, 309, 459; 142, 227, 281, 433; 142, 269, 271, 437; 213, 221, 281, 437; 213, 227, 271, 459; 213, 269, 309, 433; 221, 227, 269, $364 ; 271,281,309,364 ; 364,433,437,459 ; 48,70,74,280 ; 48,198,238,286$; $48,226,263,294 ; 48,236,245,440 ; 70,198,263,440 ; 70,226,245,286 ; 70$, $236,238,294 ; 74,198,245,294 ; 74,226,238,440 ; 74,236,263,286 ; 198$, $226,236,280 ; 238,245,263,280 ; 280,286,294,440 ; 407,94,371,118 ; 449$, $448,345,123 ; 95,322,424,440 ; 374,165,285,262 ; 493,210,317,282 ; 35$, 466, 101, 200; 499, 244, 381, 50; 350, 154, 241, 411; 114, 350, 298, 218; 231, $73,354,384 ; 227,270,236,125 ; 241,414,335,94 ; 218,37,167,308 ; 1,416$, 415,$149 ; 169,233,405,68 ; 481,326,500,113 ; 486,425,242,328 ; 145,397$, 83,$40 ; 289,352,444,497 ; 471,241,268,248 ; 94,400,279,418 ; 457,354$, 452,$133 ; 401,80,278,311 ; 131,128,386,357 ; 81,387,338,471 ; 109,446$, 93,$194 ; 137,16,215,126 ; 0,13,78,93 ; 0,17,130,391 ; 0,21,41,412 ; 0$, $29,32,501 ; 0,31,358,415 ; 0,36,81,120 ; 0,39,113,287 ; 0,51,245,432$; $0,59,83,250 ; 0,138,401,473 ; 0,117,276,439 ; 0,66,143,371 ; 0,151,349$, $461 ; 0,327,379,425 ; 0,149,208,399 ; 0,63,207,336 ; 0,70,176,275 ; 0$, 144, 301, 387; 0, 122, 323, 429; 0, 131, 265, 278; 0, 100, 259, 280; 0, 76, 192, 495; 0, 109, 134, 381; 0, 273, 343, 373; 0, 46, 218, 435; 0, 55, 108, 375; 1, 33, 109,$397 ; 0,111,272,491 ; 0,62,126,299$

For the next two theorems, we provide a lemma concerning the existence of group divisible designs with block size 4 and group type $g^{u} m^{1}$ when $g$ and $m$ are even.

Lemma 4.2. Suppose $g$ and $m$ are even, $m \equiv g(\bmod 3)$ and $g \geq 8$. Then there exists a 4-GDD of type $g^{3 t} m^{1}$ for

$$
\begin{array}{ll}
t \geq 1 \quad \text { if } \quad m=g \\
t \geq 4 \quad \text { if } \quad g<m \leq g(3 t-1) / 2, \\
t \geq 10 \quad \text { if } \quad 1 \leq m<g
\end{array}
$$

Proof. See [5] or [15, Theorem 4.6] when $m=g$; otherwise see [25, Theorem 7.1] and [13, Theorem 1.2(i)].

Although it is not needed for our purpose, if $g$ is divisible by 6 , we can improve Lemma 4.2 considerably; see [13, Theorem 1.1].

Theorem 4.1. There exists a $\mathrm{PENT}(4, r, 13)$ with deficiency graph of girth 5 for all even $r \geq 253520$. 
Proof. For $t \geq 4$, construct a $\operatorname{PENT}(4,362 t+120,13)$ with with deficiency $(13,5)$-graph using Theorem 2.5 with $3 t$ copies of the $\operatorname{PENT}(4,116,13)$ and the PENT $(4,120,13)$ from Lemma 4.1. The required 4-GDD of type $362^{3 t} 374^{1}$ exists by Lemma 4.2 .

Now take $3 u$ copies of a $\operatorname{PENT}(4,112,13)$ from Lemma 4.1 and the $\operatorname{PENT}(4,362 t+120,13)$. By Lemma 4.2 , there exists a 4-GDD of type $350^{3 u}(1086 t+374)^{1}$ provided $1086 t+374 \leq 350(3 u-1) / 2$, i.e. $u \geq(362 t+$ 183)/175. Therefore, by Theorem 2.5 , there exists a $\operatorname{PENT}(4,350 u+362 t+$ $120,13)$ with deficiency $(13,5)$-graph for $t \geq 4$ and $u \geq(362 t+183) / 175$. To show that all even $r \geq 253520$ are covered we argue as follows.

Dividing by two and ignoring the constant term, we consider

$$
\{175 u+181 t: t \geq 4, u \geq(362 t+183) / 175\} .
$$

Let $M=175 \cdot 181$ and note that $\operatorname{gcd}(175,181)=1$. By the Chinese Remainder Theorem, for any integer $m$, there exist $a_{1}$ and $a_{2}$ such that $m \equiv 175 a_{1}+181 a_{2}(\bmod M)$ with $0 \leq a_{1}<181$ and $4 \leq a_{2}<179$. By adding a multiple of 181 to $a_{1}$ whenever necessary, it follows that every integer $m$ in the range $M \leq m<2 M$ can be expressed in the form $m=175 b_{1}+181 b_{2}$ with $b_{1} \geq 0$ and $4 \leq b_{2} \leq 178=t_{\max }$.

Therefore every integer $n \geq 4 M$ has a representation of the form $n=$ $175\left(b_{1}+181 h\right)+181 b_{2}$, with $b_{1} \geq 0,4 \leq b_{2} \leq t_{\max }$ and $h \geq 2$. Put $t=b_{2}$ and $u=b_{1}+181 h$. Then $4 \leq t \leq t_{\max }$ and $u \geq 3 \cdot 181>\left(362 t_{\max }+183\right) / 175$, as required. Hence a $\operatorname{PENT}(4, r, 13)$ exists for all even $r \geq 8 M+120$.

From the proof of Theorem 4.1 we see that the upper bound of admissible $r$ for which we fail to construct a $\operatorname{PENT}(4, r, 13)$ is rather large. However, we can reduce the limit considerably if we drop the girth 5 condition and permit the use of a $\operatorname{PENT}(4,4,13)$.

Theorem 4.2. There exists a $\operatorname{PENT}(4, r, 13)$ if $r$ is even and $r \geq 474$.

Proof. Let $S=\{112,116,120, \ldots, 160\}$ and note that $S$ covers the even residue classes modulo 26 . Let $t \geq 13$.

For each $s \in S$, take $3 t$ copies of a $\operatorname{PENT}(4,4,13)$ and a $\operatorname{PENT}(4, s, 13)$, which exists by Lemma 4.1. By Lemma 4.2, there exists a 4-GDD of type $26^{3 t}(3 s+14)^{1}$. Hence, by Theorem 2.5 there exists a $\operatorname{PENT}(4,26 t+s, 13)$.

The largest value of $26 t+s$ missed by this construction is $26 \cdot 12+160=$ 472. The deficiency graphs of the constructed geometries consist of $3 t$ components $K_{13,13}$ and a 13-regular component with $3 s+14$ vertices and girth 5.

\section{Concluding Remarks}

Contrary to initial expectations, it has not been easy to find generalized pentagonal geometries where the deficiency graph has girth at least 5 and the block size exceeds 3. We have no examples of $\operatorname{PENT}(k, r, w)$ with $w>$ $k \geq 5$. Even where we have been successful the current situation is not 
entirely satisfactory in view of the considerable gap between the smallest known $(w, 5)$-graphs and the point set sizes of our geometries, as is plainly illustrated by Table 1 . For comparison, columns 3 and 5 are $2\left(w^{2}-w+1\right)$

TABLE 1. Orders of $\left(w, 5^{+}\right)$-graphs

\begin{tabular}{c|cccc} 
& $\begin{array}{c}\text { Smallest } \\
\text { known } \\
\text { PENT }(3, r, w)\end{array}$ & $\begin{array}{c}\text { Moore } \\
\text { bound } \\
\text { girth 6 }\end{array}$ & $\begin{array}{c}\text { Smallest } \\
\text { known } \\
\text { girth 5 }\end{array}$ & $\begin{array}{c}\text { Moore } \\
\text { bound } \\
\text { girth 5 }\end{array}$ \\
\hline 7 & 50,74 & 86 & $50[19]$ & 50 \\
9 & 124 & 146 & $96[20]$ & 82 \\
13 & 350 & 314 & $226[3]$ & 170 \\
15 & 514 & 422 & $310[2]$ & 226 \\
19 & 978 & 686 & $500[1]$ & 362 \\
21 & 1330 & 842 & $658[3]$ & 442 \\
25 & 2450 & 1202 & $960[3]$ & 626 \\
27 & 2794 & 1406 & $1054[3]$ & 730 \\
31 & 4298 & 1862 & $1444[3]$ & 962 \\
33 & 5134 & 2114 & $1664[3]$ & 1090
\end{tabular}

and $w^{2}+1$, the Moore bounds for $(w, 6)$ - and $(w, 5)$-graphs.

As a special case, we seem unable to create $\operatorname{PENT}(3, r, w)$ geometries with $w \in\{7,9,13,19,25,31\}$ using the incidence $(w, 6)$-graphs of $w$-GDDs of types $(w-1)^{w+1}$ and $w^{w}$. Nevertheless, from Lemmas 3.2 and 3.3 we see that $\operatorname{PENT}(3, r, w)$ geometries do exist with connected deficiency $\left(w, 5^{+}\right)$graphs and the same point set sizes, $v=2 r+w+1$, when $w \in\{7,9\}$; i.e. for $(r, w, v) \in\{(44,7,96),(45,7,98),(75,9,160),(76,9,162)\}$.

Finally, we would like to thank the referees for their comments and suggestions from which this paper has benefitted.

\section{REFERENCES}

[1] E. Abajo, G. Araujo-Pardo, C. Balbuena and M. Bendala, New small regular graphs of girth 5, Discrete Math. 340 (2017), 1878-1888.

[2] M. Abreu, G. Araujo-Pardo, C. Balbuena and D. Labbate, Families of small regular graphs of girth 5, Discrete Math. 312 (2012), 2832-2842.

[3] E. Abajo, C. Balbuena, M. Bendala and X. Marcote, Improving bounds on the order of regular graphs of girth 5, Discrete Math. 342 (2019), 2900-2910.

[4] S. Ball, J. Bamberg, A. Devillers and K. Stokes, An Alternative Way to Generalize the Pentagon, J. Combin. Des. 21 (2013), 163-179.

[5] A. E. Brouwer, A. Schrijver and H. Hanani, Group divisible designs with block size four, Discrete Math. 20 (1977), 1-10.

[6] W. Brown, On the non-existence of a type of regular graphs of girth 5, Canad. J. Math. 19 (1967), 644-647.

[7] P. J. Cameron, Combinatorics: Topics, Techniques, Algorithms, CUP, Cambridge, 1994.

[8] C. J. Colbourn, A. D. Forbes, M. J. Grannell, T. S. Griggs, P. Kaski, P. R. J. Östergård, D. A. Pike and O. Pottonen, Properties of the Steiner Triple Systems of Order 19, Elec. J. Combin. 17 (2010), \#R98. 
[9] C. J. Colbourn, D. G. Hoffman and R. S. Rees, A new class of group divisible designs with block size three, J. Combin. Theory, Series A 59 (1992), 73-89.

[10] C. J. Colbourn and A. Rosa, Triple Systems, Clarendon, Oxford, 1999.

[11] W. Feit and G. Higman, The nonexistence of certain generalized polygons, J. Algebra 1 (1964), 114-131.

[12] A. D. Forbes, Pentagonal geometries with block sizes 3, 4 and 5, J. Combin. Des. 29 (2021), 307-330; preprint: https://arxiv.org/abs/2006.15734v3.

[13] A. D. Forbes and K. A. Forbes, Group divisible designs with block size 4 and type $g^{u} m^{1}$, J. Combin. Des. 26 (2018), 519-539.

[14] A. D. Forbes, T. S. Griggs and K. Stokes, Existence results for pentagonal geometries, submitted 2020; preprint: https://arxiv.org/abs/2007.10810.

[15] G. Ge, Group Divisible Designs, Handbook of Combinatorial Designs, second edition (ed. C. J. Colbourn and J. H. Dinitz), Chapman \& Hall/CRC Press (2007), 255-260.

[16] M. Greig and R. C. Mullin, PBDs: Recursive Constructions, Handbook of Combinatorial Designs, second edition (ed. C. J. Colbourn and J. H. Dinitz), Chapman \& Hall/CRC Press (2007), 236-246.

[17] T. S. Griggs and K. Stokes, On pentagonal geometries with block size 3, 4 or 5, Springer Proc. in Math. \& Stat. 159 (2016), 147-157.

[18] H. Hanani, The existence and contruction of balanced incomplete block designs, Ann. Math. Statist. 32 (1961), 361-386.

[19] A. J. Hoffman and R. R. Singleton, On Moore Graphs with Diameters 2 and 3, IBM Journal of Research and Development 4 (1960), 497-504.

[20] L. K. Jørgensen, Girth 5 graphs from relative difference sets, Discrete Math. 293 (2005), 177-184.

[21] T. P. Kirkman, On a Problem in Combinatorics, Cambridge \& Dublin Math. J. 2 (1847), 191-204.

[22] P. Kovács, The non-existence of certain regular graphs of girth 5, J. Combin. Theory, Series B 30 (1981), 282-284.

[23] D. R. Stinson, Hill-climbing algorithms for the construction of combinatorial designs, Ann. Discrete Math. 26 (1985), 321-334.

[24] J. Tits, Sur la trialité et certains groupes qui s'en déduisent, Inst. Hautes Etudes Sci. Publ. Math. 2 (1959), 14-60.

[25] H. Wei and G. Ge, Group divisible designs with block size four and group type $g^{u} m^{1}$, Des. Codes Cryptogr. 74 (2015), 243-282.

[26] R. M. Wilson, An existence theory for pairwise balanced designs I: Composition theorems and morphisms, J. Combin. Theory Ser. A 13 (1972), 220-245.

LSBU Business School, London South Bank University, 103 Borough Road, LONDON SE1 0AA, UK

E-mail address: anthony.d.forbes@gmail.com

LSBU Business School, London South Bank University, 103 Borough Road, LONDON SE1 0AA, UK

E-mail address: c.g.rutherford@lsbu.ac.uk 